Cite this: Analyst, 2013, 138, 6032

Received 1st May 2013

Accepted 6th August 2013

DOI: 10.1039/c3an00892d

www.rsc.org/analyst

\title{
SR-FTIR imaging of the altered cadmium sulfide yellow paints in Henri Matisse's Le Bonheur de vivre (1905-6) - examination of visually distinct degradation regions $\uparrow$
}

\author{
Jennifer Mass, ${ }^{\text {*a }}$ Julia Sedlmair, ${ }^{\mathrm{b}}$ Catherine Schmidt Patterson, ${ }^{\mathrm{c}}$ David Carson, ${ }^{\mathrm{c}}$ \\ Barbara Buckley ${ }^{d}$ and Carol Hirschmugl ${ }^{\mathrm{e}}$
}

\begin{abstract}
SR-FTIR imaging has been used to map the mid-IR active photo-degradation phases in two thin sections of cadmium yellow paint removed from Henri Matisse's Le Bonheur de vivre (1905-1906, The Barnes Foundation). These samples represent both the darkened cadmium yellow foliage in the upper left of the work and the lightened cadmium yellow field beneath the central reclining figures. The altered cadmium yellow paints from both regions were found to contain cadmium carbonate $\left(\mathrm{CdCO}_{3}\right)$, cadmium sulphate $\left(\mathrm{CdSO}_{4}\right)$, and cadmium oxalate $\left(\mathrm{CdC}_{2} \mathrm{O}_{4}\right)$. Each of these phases was imaged to determine their positions as a function of depth, with the aim of better understanding the role of each phase in the degradation mechanism. This speciation mapping is critical because cadmium oxalate was used in this period as an additive in cadmium yellow light. In addition, cadmium carbonate and cadmium sulphate were synthesis starting materials for cadmium yellow, and so their distribution throughout the paint layer can provide an indication of their roles. It was established that cadmium oxalate is localized at the surface of the paint layer, cadmium carbonate is found deeper in the layer but still enriched at the surface, and cadmium sulphate is distributed throughout the layer. This distribution, along with the chloride content of the paint suggesting a cadmium chloride starting material, is consistent with an alteration mechanism in which the cadmium sulphide is oxidized to sulphate and this is then converted to carbonate and oxalate. The relative solubilities of the three photo-degradation products are also relevant to their locations in the paint film.
\end{abstract}

\section{Introduction}

The study of historic paint degradation mechanisms and alteration layers is challenging due to the fact that only microgram to milligram-sized samples can be removed from works of art in those cases where sampling is allowed. Typically these microsamples are studied by embedding them in a rapidly curing resin and polishing the resin so that the paint layers (the painting's stratigraphy) can be viewed in cross-section. Scientists may then carry out elemental and molecular microanalysis techniques in the museum laboratory such as scanning electron

${ }^{a}$ Winterthur Museum, Conservation Department, 5100 Kennett Pike, Winterthur, USA. E-mail: jmass@winterthur.org; Tel: +1-302-888-4808

${ }^{b}$ Synchrotron Radiation Center, University of Wisconsin-Madison, Stoughton, WI 53589, USA

${ }^{c}$ Getty Conservation Institute, 1200 Getty Center Drive, Suite 700, Los Angeles, USA ${ }^{d}$ The Barnes Foundation, Conservation Department, 2025 Benjamin Franklin Parkway, Philadelphia, USA

${ }^{e}$ Department of Physics, University of Wisconsin-Milwaukee, 1900 East Kenwood Blvd, Milwaukee, USA

$\dagger$ Electronic supplementary information (ESI) available: Image of calcium carbonate inclusion location versus the cadmium carbonate phase. See DOI: 10.1039/c3an00892d microscopy with energy-dispersive X-ray microanalysis (SEMEDS), microRaman spectroscopy, and attenuated total reflectance Fourier transform infrared spectroscopy (ATR-FTIR). While these tools are used routinely to identify the major phases present in paint layers, the minor and trace components of the alteration layers are commonly below the minimum detection limits of these techniques. As a consequence of these limitations, paint alteration layers are being increasingly studied using methodologies not commonly found in museum laboratories such as synchrotron radiation (SR) based techniques involving the use of X-ray ${ }^{1-3}$ and infrared microbeams. ${ }^{1,4-7}$

SR-based techniques have been successfully applied to the study of numerous pigment alteration problems, including the alteration mechanisms of cadmium sulphide yellow, ${ }^{3}$ zinc yellow, ${ }^{8}$ smalt, ${ }^{9}$ copper resinate, ${ }^{\mathbf{1 0}}$ and vivianite. ${ }^{11}$ These techniques have included spot analysis of the alteration layers using X-ray absorption near edge spectroscopy (XANES), ${ }^{12}$ SR-FTIR, ${ }^{5}$ SR-X-ray diffraction (SR-XRD), ${ }^{13}$ and SR-X-ray fluorescence (SR-XRF). ${ }^{14}$ However, recent innovations in beam focusing optics have allowed mapping of entire paint layers and paint cross-sections using techniques such SR- $\mathrm{XXR}$ mapping, ${ }^{1}$ $\mu$ XANES mapping, ${ }^{1,14}$ and SR-FTIR mapping. ${ }^{15}$ SR-based FTIR 
techniques offer improved brightness over conventional benchtop ATR imaging techniques, allowing for increased sensitivity and therefore better minimum detection limits and phase localization. IRENI SR-based FTIR imaging is also carried out in a non-contact transmission mode, which avoids the sample damage that is common if not unavoidable with ATR measurements. The measured intensities are also dependent on the amount of contact between the ATR crystal and the sample, reducing the reproducibility across an extended sampling area when the crystal must be lifted, then the sample shifted, then the crystal reengaged for sequential measurement as is necessary when measuring more than one field of view area. For advanced SR-FTIR imaging, the IRENI beamline at the University of Wisconsin Synchrotron Radiation Center in Stoughton bundles twelve SR-generated IR beams to create a unique SRFTIR source that homogeneously illuminates a multi-pixel, wide-field detector, ${ }^{16,17}$ and affords sufficient spatial oversampling to obtain diffraction-limited resolution at all wavelengths from one measurement with high signal-to-noise ratios across the spectral bandwidth.

The photo-oxidative degradation of yellow cadmium sulphide (CdS) paint dating from the 1880s to the 1920s has been observed in works by Pablo Picasso, Vincent van Gogh, Georges Seurat, Henri Matisse, Edvard Munch, James Ensor, and Ferdinand Leger. ${ }^{1,3,18,19}$ The physical manifestation of this degradation is paint chalking (drying out and crumbling), lightening, flaking, spalling (breaking off in fragments), and, in its most advanced cases, the formation of a thick ivory to tan alteration crust over the originally warm-hued yellow paint. However, the photo-oxidation begins before a colour change can be observed - a phenomenon that can be thought of as incipient photo-degradation. The chemical manifestation of this alteration is the formation of cadmium oxides, sulphates, carbonates, hydroxides, and oxalates. Detailed studies of this system in works by James Ensor ${ }^{20}$ and Vincent van Gogh ${ }^{1}$ have been carried out, and the alteration mechanism is currently being examined in Edvard Munch's The Scream (1910, The Munch Museum). ${ }^{19}$ It is important to note that the identification of oxidized cadmium phases in a cadmium yellow paint cannot, in itself, confirm that photo-degradation has taken place, since cadmium oxalate $\left(\mathrm{CdC}_{2} \mathrm{O}_{4}\right)$ was used as a filler in the preparation of the paler shades of cadmium yellow paints in this time period and cadmium carbonate $\left(\mathrm{CdCO}_{3}\right)$ was used in the same manner as a filler or precipitation substrate. ${ }^{3,21}$ In addition, both cadmium sulfate $\left(\mathrm{CdSO}_{4}\right)$ and cadmium carbonate are used as starting reagents for the synthesis of cadmium sulphide yellow paints. ${ }^{19,21}$ These factors dictate that cadmium sulphate, cadmium oxalate, and cadmium carbonate can be definitively identified as photo-degradation products only if their position as a function of depth in the paint layer is determined. If they are concentrated at the surface of the paint layer or localized in a discoloured surface alteration crust this suggests that they are photo-oxidation products.

Recent $\mu$ XANES spot analysis and mapping of cadmium yellow paints used on Henri Matisse's Le Bonheur de vivre (1905-6, The Barnes Foundation, see Fig. 1), have demonstrated that cadmium carbonate is highly concentrated in the tan alteration crust of a cadmium sulphide yellow paint (from the fruit in the tree at the upper right), suggesting its presence as a photo-oxidation product rather than a starting reagent or paint filler, but has left the role of cadmium sulphate more ambiguous. ${ }^{3}$

The $\mu$ XANES study of this material was combined with SR$\mu \mathrm{XRF}$ mapping revealing that the discolored alteration crust and visually intact (yellow) regions of the paint layer contain high concentrations of chlorine, suggesting that cadmium chloride was the starting reagent for the synthesis of the cadmium yellow rather than either cadmium sulphate or cadmium carbonate.

Previous studies have thus addressed important questions about the composition and degradation mechanisms of cadmium sulphide yellow in general and about the appearance of Le Bonheur de Vivre in particular, but they have left remaining questions about the degradation mechanisms of the darkened upper left corner of this painting (see Fig. 1), and about the presence and concentration of additional degradation products such as oxalates as a function of position. This work addresses these specific questions and related pigment degradation phenomena as examined by SR-FTIR imaging. Speciation as a function of position is critical to an understanding of the photodegradation of artists' paints, and IRENI SR-FTIR imaging has a particularly important role to play in the cadmium sulphide system given the mid-IR activity of cadmium sulphate, cadmium oxalate, and cadmium carbonate. Its molecular imaging capabilities are particularly powerful when combined with the elemental mapping capabilities of SEM-EDS and SR$\mu X R F$. IRENI FTIR imaging is used here to identify the locations of cadmium carbonate, cadmium oxalate, and cadmium sulphate in two of the most heavily altered regions of Le Bonheur de vivre, the darkened region of the work's upper left corner and the ivory-hued region below the central reclining figures (see Fig. 1).

These regions would have originally been a bright, warm yellow, based upon the comparison of the Barnes Foundation's monumental version of this work to the oil sketches owned by The Barnes Foundation, The San Francisco Museum of Modern Art, and the Statens Museum for Kunst in Copenhagen, in which the yellow paints remain unaltered. In fact, only a small percentage of the cadmium yellow paints produced between the $1880 \mathrm{~s}$ and the $1920 \mathrm{~s}$ are undergoing this degradation phenomenon. This is likely due to both the synthesis method employed to prepare each paint (there were several different manufacturers of cadmium yellow during this period and shades of cadmium yellow available on the market at the same time, and these were prepared through different synthetic routes as well as subject to different post-synthesis processing such as calcination). The inconsistent observation of this phenomenon is also likely due to the different conditions in which the paintings have been stored and displayed, including the effects of RH cycling and light exposure. However, it is notable that painters at the turn of the $20^{\text {th }}$ century such as W. H. Hunt observed that the alteration of pale cadmium yellow paints could be seen even after only 20 years $^{21}$ and so the degradation may not be a de facto result of a particular museum's environmental conditions. If this were the case, the 


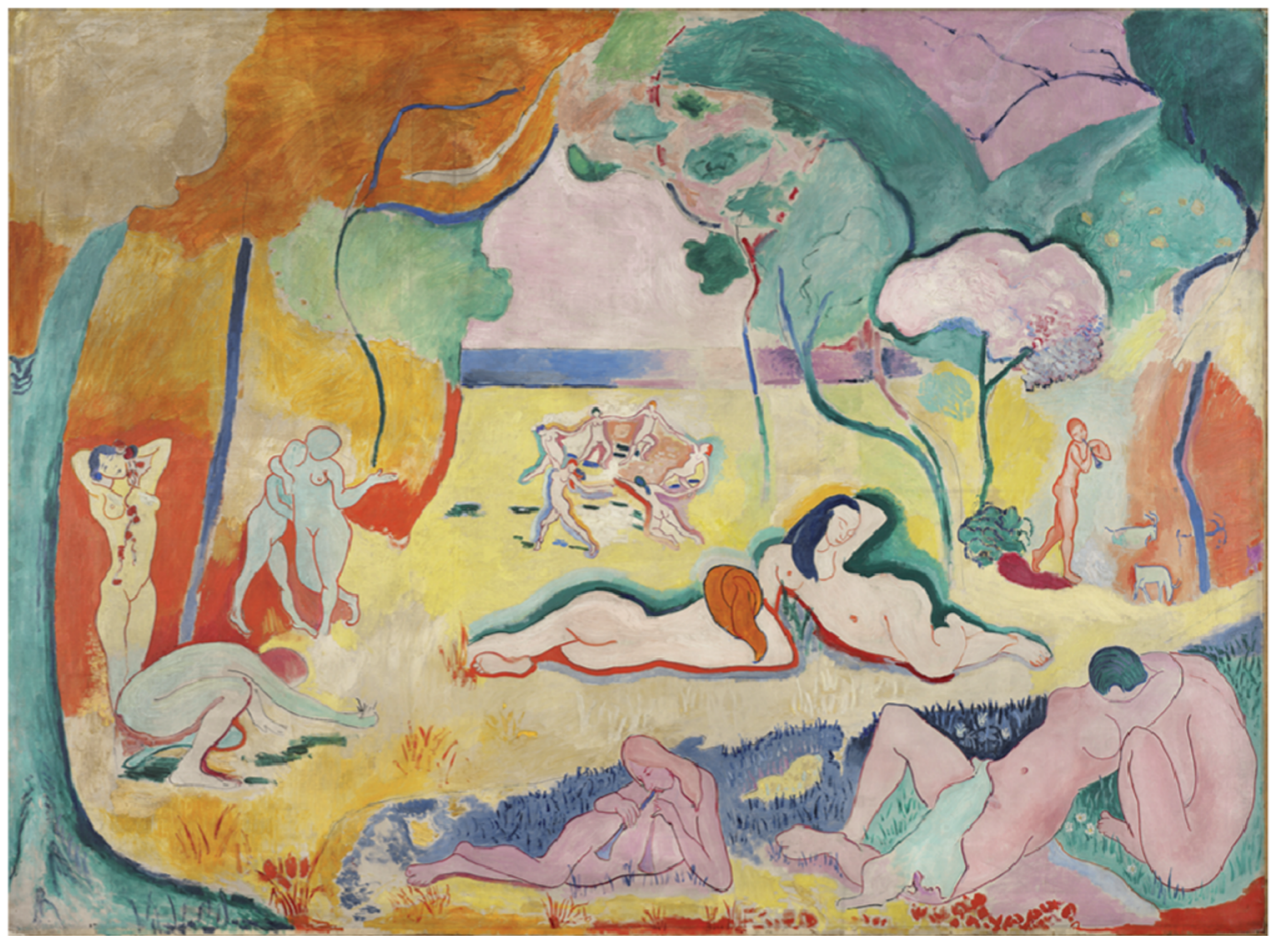

Fig. 1 Henri Matisse, French, 1869-1954 Le Bonheur de vivre, also called The Joy of Life, between October 1905 and March 1906, Oil on canvas, 69 1/2 × $943 / 4$ in. $(176.5 \times 240.7 \mathrm{~cm})$, The Barnes Foundation, BF719. Note the tan-brown alteration crusts on the yellow foliage at the upper left and the faded region below the central reclining figures.

alteration would be visible throughout certain early modernist collections, but this pattern is not observed. It is for this reason that the degradation mechanisms are of such high interest to museum scientists.

\section{Experimental}

\section{Samples analyzed}

Photo-degraded cadmium yellow paint samples were removed with a size 11 steel scalpel blade from the darkened upper left corner of the painting (sample S113) and from the faded region below the central reclining figures (sample S117). These samples were then split in two, and half were embedded in Extec polyester resin (Extec Corporation ${ }^{\circledR}$, Enfield, CT) cured with methyl ethyl ketone peroxide catalyst ( 24 hours at room temperature and under ambient light). The cubes were dry, hand-polished successively with 400- and 600-grit sandpaper and 1500- to 8000-grit MicroMesh polishing cloths to expose the cross-sections. The remaining sample portions were mounted in Bio-Plastic ${ }^{\circledR}$ Liquid Casting Plastic (Ward's Natural Science), and cured using $\sim 1 / 2$ the recommended volume of catalyst. The resulting semi-hard resin block was cut to expose the stratigraphy of the sample using an ultramicrotome (PowerTome XL, RMC Products) fitted with a diamond knife. Several individual microtome slices containing the full stratigraphy, each $\sim 3 \mu \mathrm{m}$ thick, were retained for transmission experiments on the IRENI beamline. The intrinsic friability of these two samples causes unacceptable levels of material loss at section thicknesses below $3 \mu \mathrm{m}$.
Softened Bio-Plastic resin was used for the IRENI samples because it provides an appropriate combination of longevity and ease of transport of the thin sections, environmental stability, and appropriateness to the technique of microtoming small, irreplaceable samples. Although the resin itself may infiltrate the sample and has a mid-IR signature, spectral interferences can be avoided by judiciously selecting the specific bands to image for the oxalates, carbonates, and sulphates being measured.

While this mounting technique cannot overcome the inherent friability of the samples (the extent of which cannot easily be determined prior to mounting), it affords distinct advantages over other mounting techniques available. For example, although mounting in $\mathrm{KBr}$ eliminates interferences in the mid-IR spectral region, the hygroscopic and brittle nature of $\mathrm{KBr}$ does not allow the sample to be retained and re-examined over time with additional techniques, such as full field XANES imaging. This type of re-use is often necessary when the samples are precious as in the case of fine art materials. Challenges encountered when using $\mathrm{AgCl}$ and $\mathrm{BaF}_{2}$ pellets include obtaining and retaining the proper mounting orientation due to the opacity of the salts, and potential for crushing the paint cross-section during pellet pressing (Pouyet et al. this volume), ${ }^{22}$ compromising stratigraphic data. Epoxy resins are far too hard for microtoming brittle paint cross-sections and result in extensive sample crumbling.

Barrier methods that may help reduce or eliminate sample impregnation by the mounting resin are also challenging. Using gold or aluminium foils around the paint cross-section 
increases the risk of sample loss during microtoming because the sample is not visible. While the use of cyclododecane avoids this risk, the sublimation of the cylcododecane after sectioning leaves a brittle and friable section that is too delicate to manipulate, and the procedure involves exposing the sample to a solvent and to a curing step at $58{ }^{\circ} \mathrm{C}$ that may induce chemical change in the sample. Pouyet et al. ${ }^{22}$ (this volume) have addressed the preparation of thin sections of historic paint cross-sections with minimal analytical interferences. These methods have included using a diamond micro-compression cell, which can provide high quality data, but can also distort or destroy the stratigraphy of the sample. Sample enclosing systems employed at ESRF involving polycarbonate film encapsulation and $\mathrm{AgCl}$ resin embedding have shown promise, but are still being developed.

\section{Museum-laboratory analysis}

Cross-section samples prepared in Extec were used for photomicroscopy in visible and ultraviolet illumination followed by elemental mapping using SEM-EDS. Photomicroscopy was carried out using a Nikon $80 \mathrm{i}$ microscope with a $10 \times$ ocular and a $20 \times$ objective $\left(200 \times\right.$ total magnification), the $\mathrm{X}$-cite ${ }^{\circledR} 120$ fluorescence illumination system, and ultraviolet filter cube BV2A which has a 400-440 nm excitation. Act-1 software was used to collect the images, shown in Fig. 2 and 3. Photomicroscopy of IRENI transmission samples was carried out using a Leica DM4000 microscope with both visible and UV illumination. A $10 \times$ ocular, and $20-100 \times$ objectives $(200-1000 \times$ total magnifications), and ultraviolet filter cube A, which has a $340-380 \mathrm{~nm}$ excitation filter, were utilized. The Spot software suite was used to collect images. Visible and ultraviolet photomicrographs of samples S113 and S117 are shown immediately following preparation in Fig. 2 and 3 respectively.

The block-face samples mounted in Bio-Plastic ${ }^{\circledR}$ Liquid Casting Plastic were further examined using scanning electron microscopy with energy-dispersive X-ray microanalysis (SEMEDS) to provide elemental information about each section. The SEM-EDS system used was a FEI-Philips XL30 ESEM-FEG with an Oxford Inca EDS system. All maps were run at $20 \mathrm{keV}$ with spot size 3. Backscattered electron (BSE) images of the two sections are also shown in Fig. 2 and 3.

\section{SR-FTIR}

SR-FTIR imaging of the thin section samples was performed using the IRENI beamline. The IRENI beamline is unique in the field of synchrotron-based FTIR imaging because it enables wide-field, high resolution infrared (IR) imaging with the high throughput and signal-to-noise produced by a bright, stable synchrotron beam. It provides new capabilities that can address some of the challenges related to the analysis of small, multilayered cross-section samples, as was demonstrated in previous work utilizing sample S117. ${ }^{23}$

Like other SR-FTIR methods, FTIR imaging at the IRENI beamline is typically conducted in transmission mode, eliminating the spectral artefacts observed in ATR-FTIR spectra that often result in the need for additional data processing. Unlike other SR-FTIR mapping methods, the IRENI beamline allows for the collection of diffraction-limited resolution at all wavelengths simultaneously, allowing for detailed analysis of the smallest samples, as well as high signal to noise across much of the mid-infrared region. Note that for IRENI SR-FTIR measurements diffraction-limited resolution arises from the limitation that far-field resolution is limited by physical optics, and is wavelength dependent. ${ }^{17}$ This Rayleigh Criterion is typically quoted as $d=.61 \lambda / \mathrm{NA}$, where $d$ is the distance between two objects, $\lambda$ is the wavelength of the light, and NA is the numerical aperture of the objective. Since IR microscopes use Schwarzschild objectives, the relationship is similar but not available in a simple closed form. ${ }^{24}$

ATR-FTIR mapping, specular reflection FTIR mapping, and SR-FTIR mapping have been used by Boon et al. to study the formation of metal carboxylate aggregates in paint films and to characterize the deterioration of bone black pigment in paint cross-sections. ${ }^{25-28}$ While these methods offer detailed chemical maps, the IRENI beamline offers pixel sizes of down to $0.54 \times$ $0.54 \mu^{2}$ which is sufficiently spatially oversampled to allow for higher resolution at all wavelengths, providing more detailed images representing true chemical imaging rather than raster
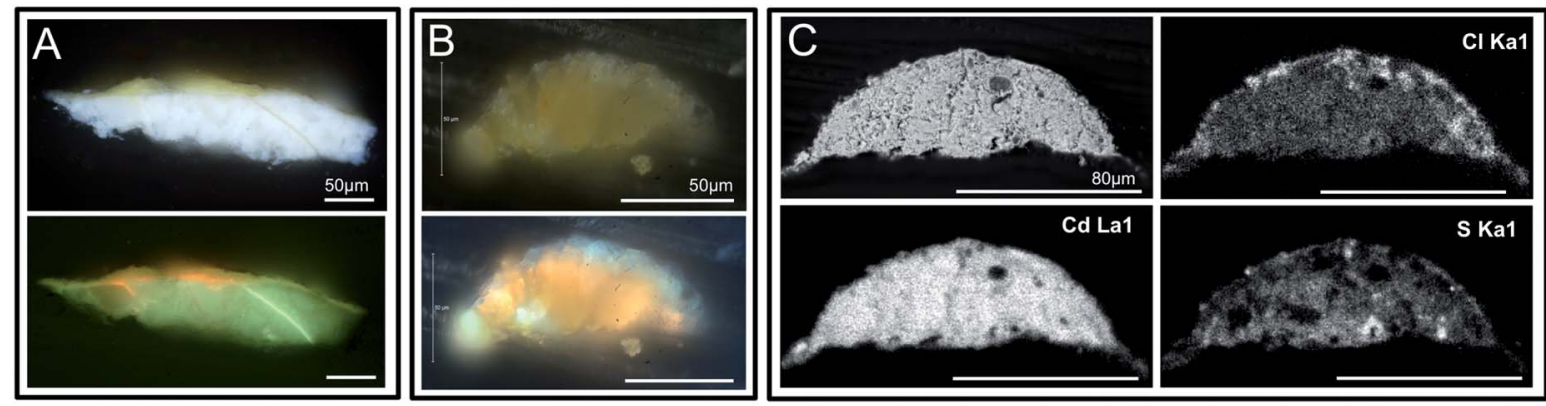

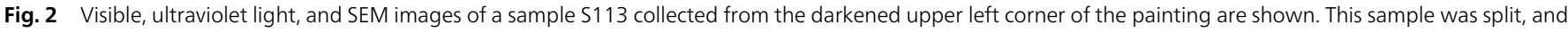

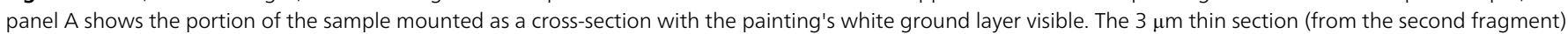

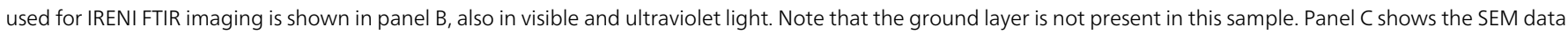

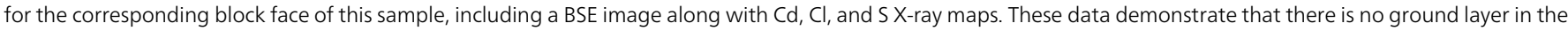

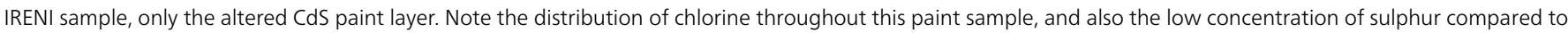
cadmium. 

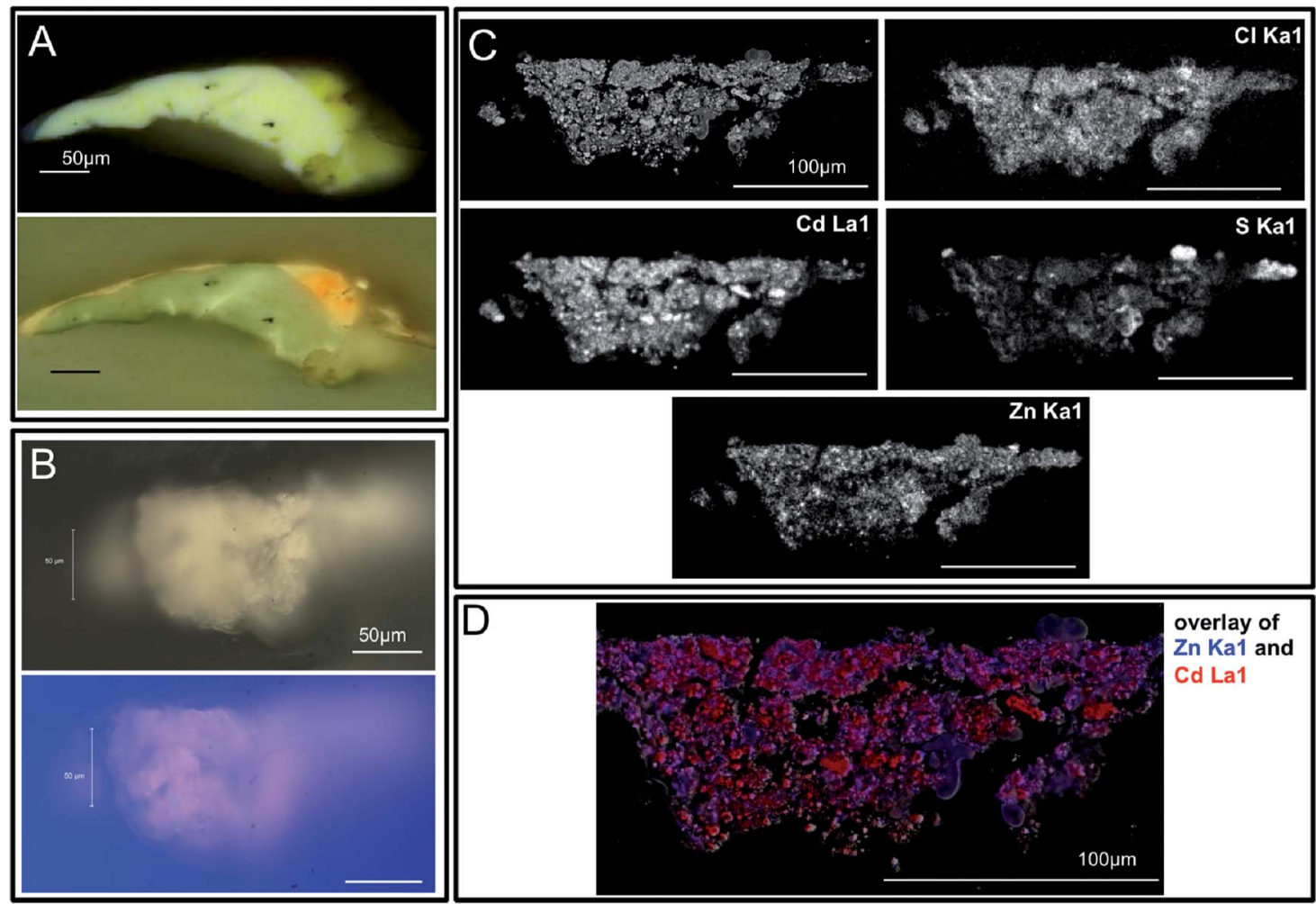

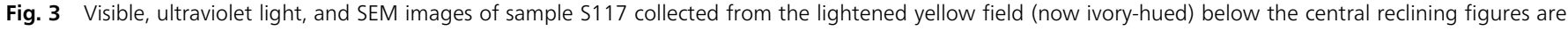

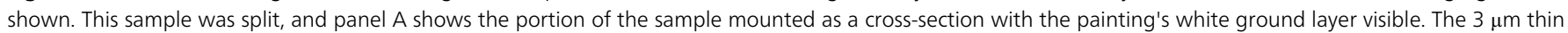

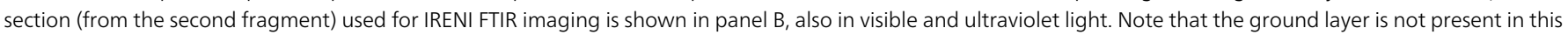

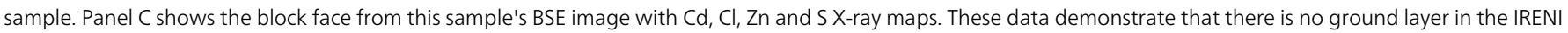

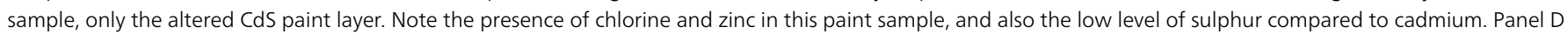
shows the locations of $\mathrm{Cd}$ and $\mathrm{Zn}$ in this sample in red and blue respectively. Note that the $\mathrm{Zn}$ appears to be present as a separate phase, likely ZnO.

scanning, which relies upon setting an aperture that defines the spatial resolution of the final images. Kazarian et al. have carried out diffraction-limited FTIR imaging using a laser-based tabletop IR imaging system that, while it offers diffractionlimited spatial resolution, has a spectral resolution substantially below the IRENI method $\left(<20 \mathrm{~cm}^{-1}\right) .{ }^{29}$ While specular reflection geometry measurements are compatible with traditional sample mounting techniques, they are totally dependent on the heterogeneous nature of the material that is being evaluated. The electric field penetrates much smaller distances for higher absorbing regions than lower absorbing regions. By definition for a heterogeneous material the measurement at each pixel will be entirely dependent on what material is encountered in the top layers of the sample, and will penetrate varying depths of the sample, which would be challenging, if not impossible, to evaluate accurately.

For the analysis using IRENI, prepared samples were supported on $\mathrm{a} \mathrm{BaF}_{2}$ window which was also used to collect background measurements. The beamline was used as typically configured for transmission measurements: a $74 \times / 0.6$ NA Schwarzschild objective (Ealing) and $15 \times / 0.58$ NA Schwarzschild condenser (NicPlan) on a commercial IR microscope (Bruker Vertex 70 FTIR/Hyperion 3000 microscope) equipped with a $128 \times 128$ pixel $\mathrm{HgCdTe}$ focal plane array detector (FPA,
Santa Barbara Focalplane, range of $3950-950 \mathrm{~cm}^{-1}$ ). This experimental setup provides an effective geometrical pixel area at the sample plane of $0.54 \times 0.54 \mu^{2}$. A $64 \times 64$ pixel area of the detector was illuminated and recorded as a tile. Thus, each tile used to build images of the samples contains 4096 individual spectra and images an area of $\sim 34 \times 34 \mu \mathrm{m}^{2}$. Data were collected using the commercial OPUS software suite (Bruker) using 256 sample scans and an integration time of $0.10475 \mathrm{~ms}$, and analyzed using IRidys, a program for the commercial software package IGOR Pro, which was generated in-house at SRC.

\section{Results and discussion}

Both historic mineral-based pigments and modern synthetic pigments used in painting are susceptible to alteration as a result of their reactions with light, atmospheric moisture, oxygen, sulphur dioxide, and other agents of degradation in their environment. Modern synthetic pigments that suffer degradation include the nineteenth-century yellow pigments chrome yellow ${ }^{30-33}$ and zinc yellow ${ }^{8,34}$ in addition to cadmium yellow. ${ }^{\mathbf{1 1 8 - 2 0}}$ The degradation mechanisms behind these alterations are often complex, involving traces of synthesis starting reagents or other impurities in the paint, requiring the presence of multiple degradation agents (e.g. light and high relative 
humidity) or triggered by the alteration of a neighbouring pigment. The study of these complex phenomena is hindered by the fact that they are often occurring within only the uppermost layers of the paint film that is itself only several micrometers thick (artists' paint layers can vary from as little as three micrometers thick to as large as hundreds of micrometers thick, with approximately twenty micrometers being the average for a single layer of brush-applied oil paint). Thus the alteration layer can be only 1-2 micrometers thick or, depending on the state of degradation, can extend throughout an entire paint layer.

Fig. 2 and 3 reveal that the samples characterized using IRENI SR-FTIR imaging contain the altered cadmium-containing paint film only, and none of the painting's ground layer. This is critical since the ground is composed of clastic barium sulphate particles in a lead white (hydrocerussite) matrix, and thus would contribute to the carbonate and sulphate signals being studied. The lack of this ground layer in the studied samples means that the carbonate and sulphate signals can be reasonably assumed to stem from the degradation of the parent cadmium sulphide pigment. Fig. 2 shows the inhomogeneous distribution of sulphur in the paint layer of sample S113, with the surface of the sample being particularly depleted in this element. Chlorine is distributed throughout the paint layer, with its highest concentration at the surface of the paint layer. The presence of chlorine throughout this sample raises the possibility that $\mathrm{CdCl}_{2}$ was the starting material for the synthesis of the cadmium sulphide. It has been shown that CdS synthesized from $\mathrm{CdCl}_{2}$ has a lower band gap than CdS synthesized from other starting reagents, and absorbs light across a larger region of the visible spectrum. As a result, it has the potential for greater photo-induced reactivity than other sources of the same pigment. ${ }^{35}$ The thin films for which these phenomena were measured have a CdS grain size range from 2.9 to 3.9 nanometres. Similarly, uncalcined precipitated CdS prepared in our laboratory was found to have an average particle size of 2.9 nanometres. Cadmium sulphide yellow pigments from the 1880 s through the 1920 s were often uncalcined, and as a result nanocrystalline or amorphous, which can be observed by their lack of an XRD pattern. ${ }^{18}$ No zinc map was included for this sample as it was essentially zinc-free, in contrast to S117.

Fig. 3 shows the depletion of sulphur relative to cadmium in sample $\mathrm{S} 117$, the sample removed from the lightened cadmium yellow paint below the central reclining figures. Chlorine is distributed throughout the paint layer here too, suggesting that $\mathrm{CdCl}_{2}$ may also have been the starting material for the cadmium yellow paint in this region. However, the high concentration of zinc throughout the paint layer suggests that a different tube of cadmium yellow may have been used for this region of the painting than was used for the region at the upper left. While cadmium is mined from zinc ores (and so cadmium yellow paints frequently contain trace or minor amounts of zinc), the concentration of zinc here is more suggestive of a zinc oxide added by the manufacturer or artist supplier. The addition of zinc oxide - a white pigment - to the cadmium yellow paint in this region appears to be consistent with Matisse's oil sketch now in the collection of the San Francisco Museum of Modern Art [Henri Matisse, Sketch for Le Bonheur de vivre (Joy of Life,), oil on canvas $16 \times 21 \frac{1}{2}$ in. $(40.64 \times 54.61 \mathrm{~cm})$, SFMOMA 91.160], in which Matisse also chose a lighter yellow under the central figures and a darker, more orange-hued yellow for the upper left tree foliage.

In Fig. 4, reference spectra for cadmium carbonate, cadmium sulphate, cadmium oxalate, and the Bio-Plastic ${ }^{\circledR}$ mounting resin are shown along with an average spectrum for each sample. The sample spectra are calculated averages from all of the pixels acquired during imaging of each sample $>24500$ spectra in the case of sample 113, and 81920 spectra in

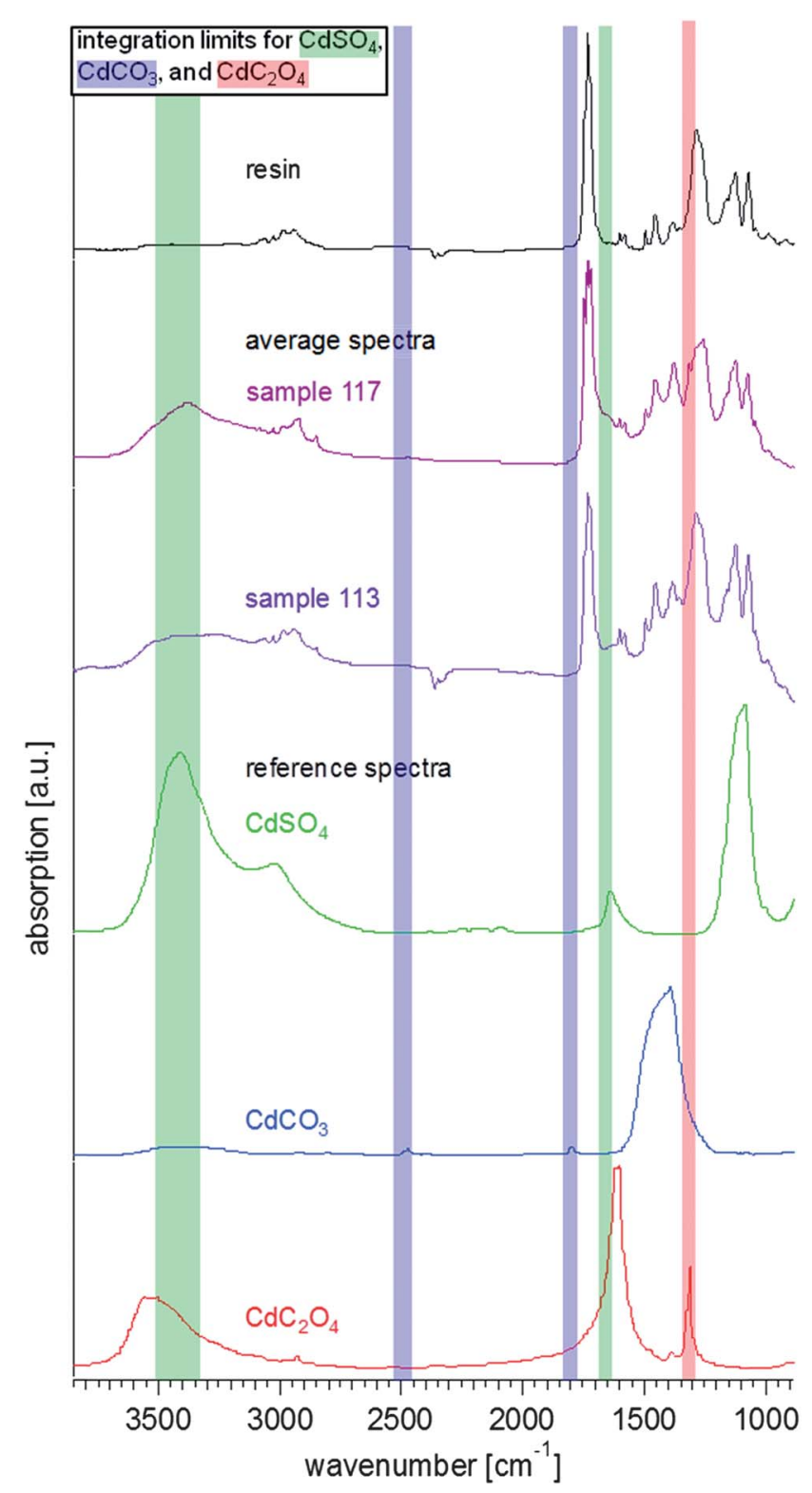

Fig. 4 Reference and average FTIR spectra from the measured samples. The average spectra (extracted from multiple pixels of the hyperspectral data sets for each sample) show the resin (taken from the resin surrounding S113, black), and the results from $\mathrm{S} 113$ (purple) and $\mathrm{S} 117$ (violet). The reference spectra of cadmium sulphate $\left(\mathrm{CdSO}_{4} \cdot n \mathrm{H}_{2} \mathrm{O}\right.$, Sigma-Aldrich, in green), cadmium carbonate $\left(\mathrm{CdCO}_{3}\right.$, in blue) (Sigma-Aldrich), and cadmium oxalate $\left(\mathrm{CdC}_{2} \mathrm{O}_{4} \text {, in red }\right)^{36}$ are stacked in the lower half of the graph. The integration limits for the chemical images in Fig. 5-8 are also highlighted in the same colour code. 
the case of sample 117 . The mounting resin reference spectrum is taken from a separate area of sample S113, and so is also a calculated average from all of the pixels within the field of view, $>24500$ spectra. Fig. 4 demonstrates the potential overlaps and distinguishing spectral bands for this limited set of materials of interest. Note that the samples are complex mixtures, and likely contain many other materials [such as the paint binding medium (a drying oil) and possible restoration materials], which is why the average spectra also show more complex signatures. These average spectra also include regions containing resin only (see ESI $\dagger$ ).

To determine the presence/distribution of each constituent of interest in the samples, a peak whose area can be integrated must be identified, and these integration regions must be chosen with care to minimize spectral interferences during imaging. Fig. 4 also shows highlighted spectral ranges that indicate the integration regions chosen for each of the cadmium-containing species. We have chosen two absorption bands for cadmium carbonate that are clearly separated from the other spectral features in these reference materials (indicated in blue). These are weak absorbers, but since they are distinctive and separated from the fingerprint region (1650-900 $\mathrm{cm}^{-1}$ ) where there is substantial overlap with the mounting resin and the paint binder, they are more reliable than, for example, the substantially more intense asymmetric carbonate stretching band near $1427 \mathrm{~cm}^{-1}$. The two bands that have been chosen for cadmium sulphate (indicated in green) overlap somewhat with two absorption bands for cadmium oxalate (in red), which suggests that care is needed in interpreting these bands. In order to address this potential overlap, the spectral bandwidth and integration parameters for both absorption bands have been limited at the peak of the band, with a corresponding baseline. The band at $1314 \mathrm{~cm}^{-1}$ for the oxalate is very sharp, and distinctive, even though it is in the middle of the fingerprint region. The average spectra for both samples clearly show evidence that most of these compounds are present in the samples - noting the broad nature of the band at $3400 \mathrm{~cm}^{-1}$ for the sulphate with the clear shoulder at high frequency that aligns well with the oxalate band, and the sharp band at 1314 $\mathrm{cm}^{-1}$ for the oxalate. Interestingly, the weak bands for the carbonate at 1750 and $2450 \mathrm{~cm}^{-1}$ are only visible in the average spectrum for sample 117 , even though they fall within a region of little absorption from other constituents. These bands are very clear in average spectra for specific regions of interest, as will be discussed below. This result suggests that if one were to collect only one average spectrum for the entire sample, one could miss important phases present.

In Fig. 5 and 6, visible images (Panels A) of the thin sections for sample 113 and 117 that were measured with IRENI are shown, each with four integrated intensity IR images and the former with five accompanying spectra. Note that the top surface of each paint fragment is always at the top of the SR-FTIR image (i.e., each sample is right-side up). The visible image of sample 113, shown in Fig. 5A, shows damage that occurred to the prepared sample in transit to the beamline some of the inorganic pigment has fallen away from the centre of the sample, leaving a hole in the sample. The chemical images are generated from integrating the individual spectra at each pixel for a given spectral range, using the integration regions shown in Fig. 4. Panels B (with ROI1) and C (with ROI2) represent the strength of $\mathrm{CO}_{3}{ }^{2-}$ (carbonate) functional group absorption bands (2429-2510 $\left.\mathrm{cm}^{-1}, 1777-1816 \mathrm{~cm}^{-1}\right)$ for cadmium carbonate. Panels D (with ROI3) and E (with ROI4) represent the strength of $\mathrm{OH}$ (from waters of crystallization) absorption bands (3333-3504 $\mathrm{cm}^{-1}$, and sulphate bending band $1624-1672 \mathrm{~cm}^{-1}$ ) for cadmium sulphate. The colour scales are rainbow colour scales (violet and magenta low, red high). For Fig. 5 only, average spectra from the combined pixels in each of the four circular or elliptical regions of interest (as labelled in the panels) are shown, with highlighted spectral ranges for clarity. The double highlight indicates which band was integrated for the corresponding image. The significance and detailed analysis of these FTIR images is discussed separately for each sample below.

Further inspection of the spectra for the regions of interest is warranted. In all of these spectra it is clear that multiple constituents are contributing to the spectra; however there is a minor contribution from the oxalate.

\section{Phase distributions and relationships to alteration mechanisms}

As noted above, the identification of photo-degraded cadmium sulphide is challenging because two of the most commonly observed photo-degradation products, cadmium carbonate and cadmium sulphate, are synthesis starting reagents that may be found as impurities in cadmium yellow paints. A third photodegradation product, cadmium oxalate, is thought to have been used as an inert filler/extender in paler shades of cadmium yellow paints, as was cadmium carbonate (although in this case the synthesis conditions may have resulted in the co-precipitation of the white cadmium carbonate with the cadmium sulphide thus producing a paler shade). ${ }^{19}$ These challenges make it necessary not to immediately interpret the presence of cadmium carbonate, sulphate, and oxalate in cadmium yellow paints as photo-degradation products (even though they are commonly observed in photo-degraded cadmium yellow paints). To understand the origin and role of cadmium carbonates, sulphates, and oxalates in these altered paint layers, then, it is necessary to understand the concentration of these phases as a function of depth.

Fig. 5 of sample S113 from the darkened upper left corner shows cadmium carbonate is concentrated at the surface of paint cross-section. This phenomenon has been observed previously using $\mu$ XANES analysis in the degraded cadmium yellow fruits of the tree at Le Bonheur de vivre's upper right, which have a similar mottled tan alteration surface. ${ }^{14}$ Cadmium carbonate's location at the surface suggests that it is present in the sample, at least in part, as a photo-degradation product. The high concentrations of chloride in this paint layer (see Fig. 2), however; suggest that cadmium chloride rather than cadmium carbonate was the starting synthesis reagent for the cadmium yellow pigment observed here. Cadmium carbonate has also been observed in photo-degraded regions of Edvard Munch's 

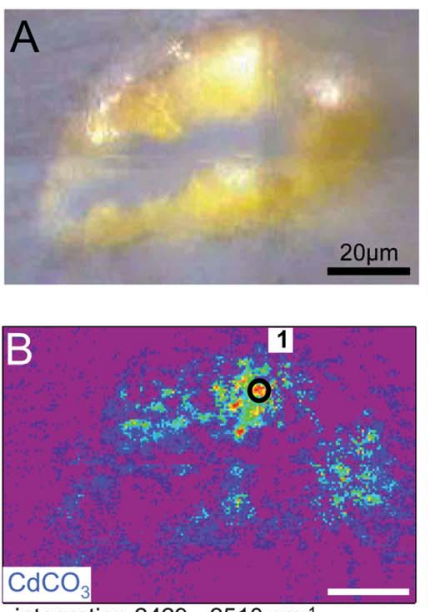

integration 2429 - $2510 \mathrm{~cm}^{-1}$

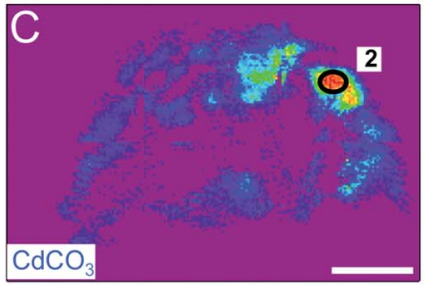

integration 1777 - $1816 \mathrm{~cm}^{-1}$
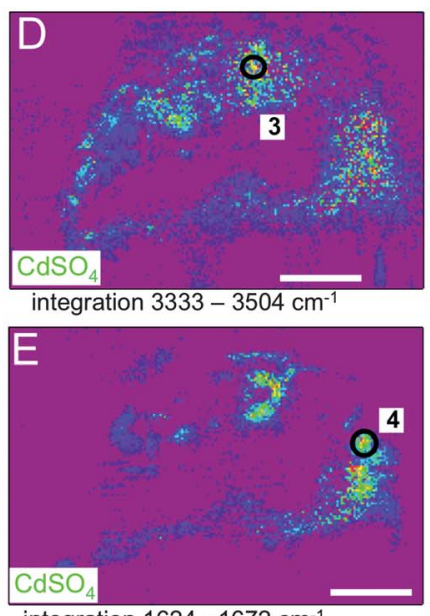

integration 1624 - $1672 \mathrm{~cm}^{-1}$

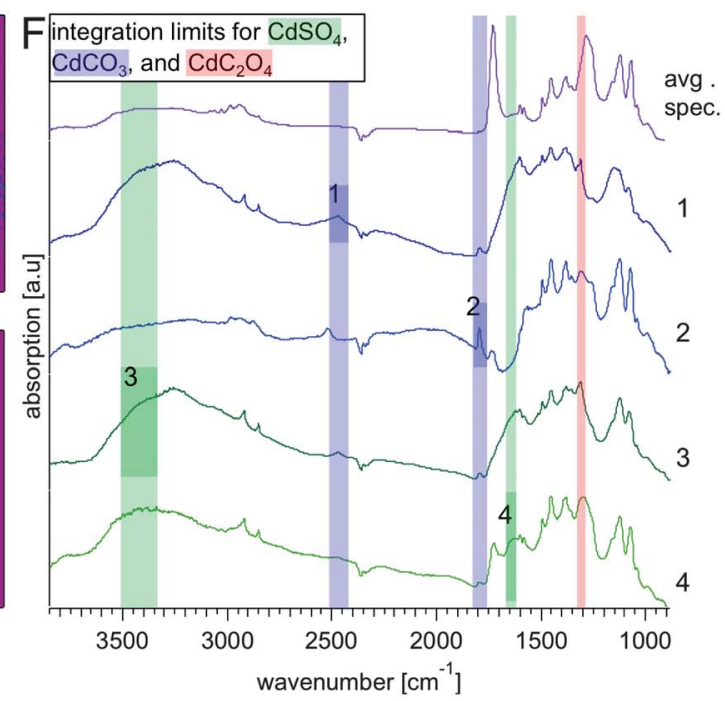

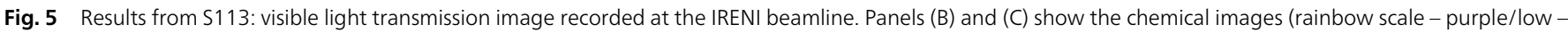

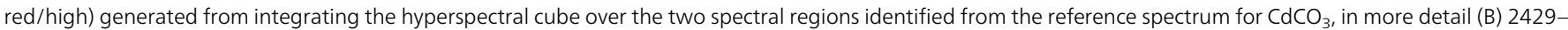

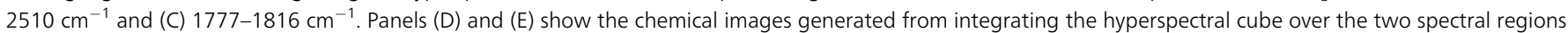

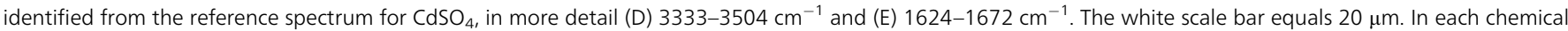

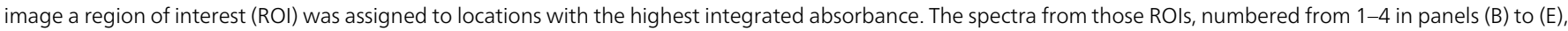

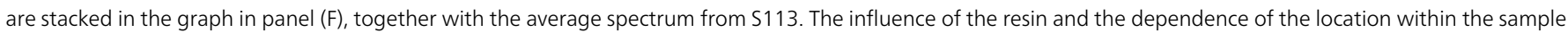
are clearly visible.

The Scream (Munch Museum 1910), ${ }^{19}$ in Vincent van Gogh's Flowers in a Blue Vase (1887 Kröller-Müller Museum), ${ }^{1}$ as well as in works by Jessica Heath (The Daffodil Fields, 1920, Private Collection) and Pablo Picasso Flowers (1901, The Tate Modern). ${ }^{18}$ The location of the $\mathrm{CdCO}_{3}$ in the paint layer as a function of depth has only been studied in the case of Le Bonheur de vivre (described above) and in Vincent van Gogh's Flowers in a Blue Vase. $\mathrm{CdCO}_{3}$ is present in altered yellow paint samples in Flowers in a Blue Vase in both the alteration zone (also containing oxalates) as well as throughout the yellow paint layer, suggesting that it may play a dual role as a residual starting reagent or filler as well as a photo-degradation product. ${ }^{1}$ Mechanisms for the formation of $\mathrm{CdCO}_{3}$ have been discussed by Mass et al. ${ }^{\mathbf{1 4}}$ as well as Van der Snickt et al., ${ }^{1}$ with possible cadmium sulphate and cadmium oxalate intermediates. Further mechanistic research, likely involving artificial aging of model systems, will be required to fully address this question, as noted by Van der Snickt et al.

Interestingly, for ROI 2 in this sample (see Fig. 5C), where the $1387 \mathrm{~cm}^{-1}$ and $1700 \mathrm{~cm}^{-1}$ carbonate bands appears strongly, the $2500 \mathrm{~cm}^{-1}$ band is shifted. This shift to a peak maximum of $2518 \mathrm{~cm}^{-1}$ suggests that $\mathrm{CaCO}_{3}$ (calcite or aragonite form) could be present in this region rather than $\mathrm{CdCO}_{3}\left(\mathrm{CaCO}_{3}\right.$ has a peak maximum at $2512 \mathrm{~cm}^{-1}$ while $\mathrm{CdCO}_{3}$ has one at $2470 \mathrm{~cm}^{-1}$ ). Elemental mapping on the corresponding block face to this thin section shows a calcium hotspot that provides confirmation of this finding, critical since $\mathrm{CaCO}_{3}$ has not been observed in the cadmium yellow paints of Le Bonheur de vivre previously. It is also notable that there is a smaller concentration of sulphates and oxalates in this region than elsewhere in the sample, supporting the possibility that this is a carbonate inclusion unrelated to the CdS photo-degradation (see ESI $\dagger$ ).

The distribution of cadmium sulphate in sample S113 can also be observed in Fig. 5. Unlike cadmium carbonate, cadmium sulphate can be observed throughout the paint layer, suggesting either that it is present as a residual synthesis reagent or that it is a more mobile photo-oxidation product. As noted by Van der Snickt et al., cadmium sulphate is highly soluble (cadmium sulphate monohydrate has a $K_{\mathrm{sp}} 76.7 \mathrm{~g} / 100$ $\mathrm{mL}$ at $25^{\circ} \mathrm{C}$ ), in comparison to cadmium carbonate (a $K_{\mathrm{sp}}$ of $\left.1.0 \times 10^{-12}\right)$. As cadmium sulphide itself is insoluble $\left(K_{\mathrm{sp}}\right.$ of $1 \times$ $10^{-27}$ in water at $25^{\circ} \mathrm{C}$ ), it is clear that any photo-oxidation to cadmium sulphate at the surface of the paint film would not constrain the cadmium sulphate to a surface-based alteration layer in the same way that the formation of the less soluble $\mathrm{CdCO}_{3}$ and $\mathrm{CdC}_{2} \mathrm{O}_{4}$ compounds might (solubility product of $\mathrm{CdC}_{2} \mathrm{O}_{4}$ is $\left.1.42 \times 10^{-8}\right) .{ }^{1,37}$ This would especially be the case in a high relative humidity environment, where moisture and soluble salts could move together through the paint structure. This same phenomenon of widespread sulphate distribution 

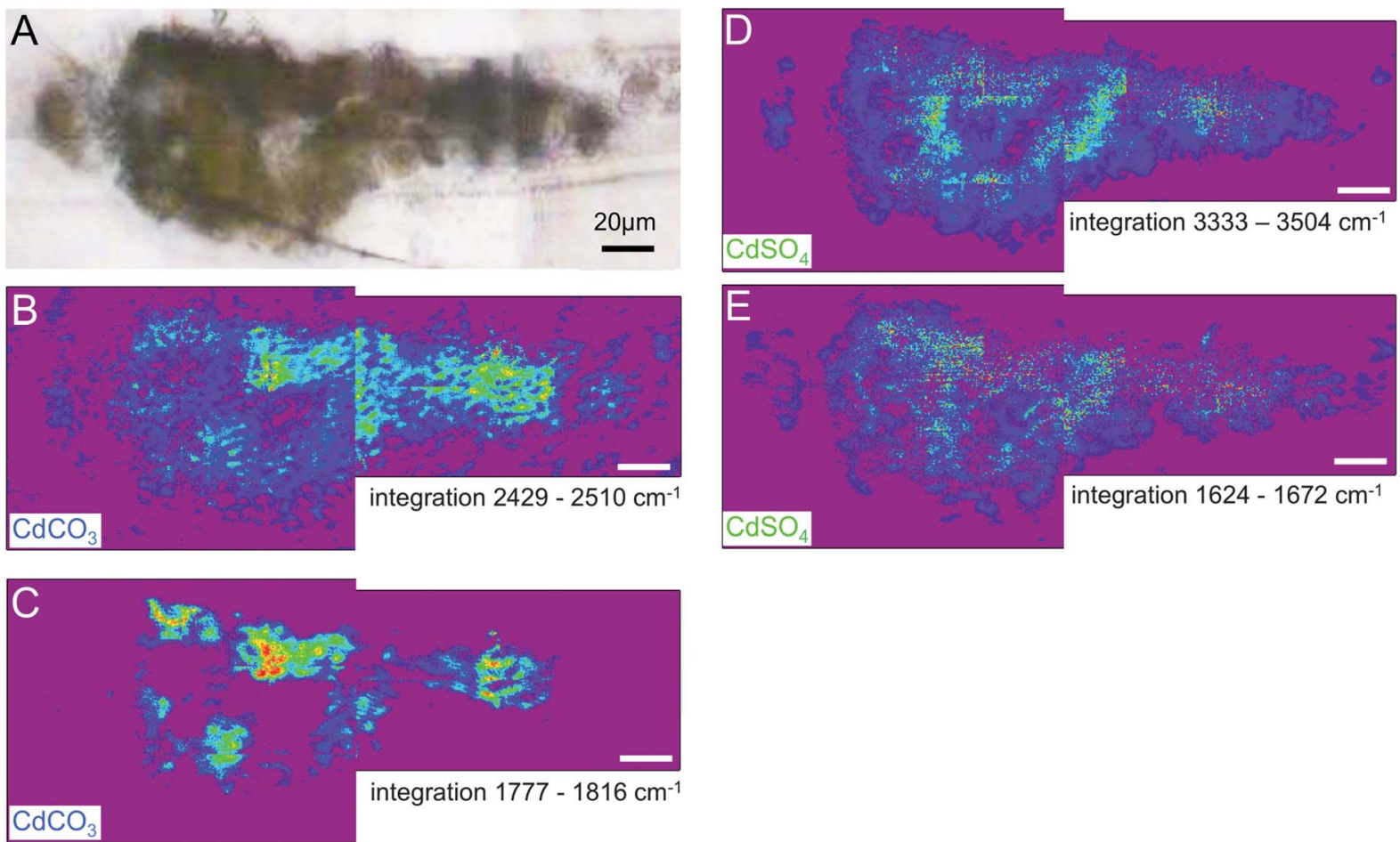

Fig. 6 Results from S117: (A) visible light transmission image recorded at the IRENI beamline. Chemical images analogous to the results presented in Fig. 5 for S113 are shown. The chemical images (rainbow scale - purple/low - red/high) show the distribution of $\mathrm{CdCO}_{3}$ in (B) $2429-2510 \mathrm{~cm}^{-1}$ and (C) $1777-1816 \mathrm{~cm}^{-1}$ and the distribution of $\mathrm{CdSO}_{4}$ in (D) $3333-3504 \mathrm{~cm}^{-1}$ and (E) $1624-1672 \mathrm{~cm}^{-1}$. The white scale bar is $20 \mu \mathrm{m}$.

was previously observed using $\mu$ XANES on a sample from one of the cadmium yellow fruits in the tree at the upper right. ${ }^{14}$ In this instance, regions of the tan alteration crust contained over $90 \%$ $\mathrm{CdCO}_{3}$. Due to the sample failure in the middle of the sample S113, exact comparisons of the concentration of cadmium sulphate in the bulk and at the surface are challenging. However, slight sulphate enrichment was previously detected on the very surface of sample S113 using $\mu$ XANES mapping, a technique with higher spatial resolution, and this result is consistent with the locations of the red "hot spots" indicating highest sulphate concentration on the FTIR image. ${ }^{\mathbf{1 4}}$ This surface enrichment of cadmium sulphate has also been observed in sample 55 , below the central reclining figures, using $\mu$ XANES mapping, suggesting that while $\mathrm{CdSO}_{4} \cdot n \mathrm{H}_{2} \mathrm{O}$ has the potential solubility to migrate through the paint layer, that some surface accumulation resulting from photo-degradation can still be observed. ${ }^{14}$

Fig. 6 shows the distribution of cadmium carbonate and cadmium sulphate in sample S117, a sample removed from an island of yellow paint beneath the central reclining figures. Cadmium carbonate appears to be present in highest concentrations at or near the top of the paint layer based on the 2429$2510 \mathrm{~cm}^{-1}$ integration image (Panel B). While this trend is not as evident in the $1777-1816 \mathrm{~cm}^{-1}$ integration image (Panel C), all of the red "hot spots" of highest concentration occur toward to the top surface of the sample, suggesting cadmium carbonate's role as a photo-degradation product. This finding is consistent with the SEM-EDS data on this sample, which reveals depletion of sulphur toward the surface of the paint layer, with the exception of two crystals above the surface of the paint layer, which appear euhedral, and may represent a crystalline sulphur-containing degradation product (see Fig. 3). While these crystals did not survive transport to the IRENI facility, the increased translucency of these crystals, and the other sulphurrich regions of the SEM-EDS map, is consistent with the formation of a hydrated sulphate salt. The cadmium carbonate integration images using the $2429-2510 \mathrm{~cm}^{-1}$ regions and the $1777-1816 \mathrm{~cm}^{-1}$ regions do show some carbonate intensity throughout the paint section (although again highest intensities are observed at or near the painting's surface). This is consistent with $\mu$ XANES data of sample S115 from the yellow fruit of the tree at the upper right, which reveal that in the bottom (and still yellow) portion of the paint sample as much as $46 \% \mathrm{CdCO}_{3}$ can be observed. These findings can be attributed to the increased solubility and thus mobility of $\mathrm{CdCO}_{3}$ over CdS as the $\mathrm{CdCO}_{3}$ photo-degradation product forms, or as being suggestive of the presence of a $\mathrm{CdCO}_{3}$ residual synthesis starting reagent or filler. Given that the high concentration of chloride in this sample suggests a $\mathrm{CdCl}_{2}$ synthesis starting reagent, however, the latter explanation seems less likely.

Panels D and E show the distribution of cadmium sulphate throughout this sample. Similar to sample S113, the sulphate is widely distributed throughout the paint cross-section. This is consistent with the high solubility (and thus mobility) of this phase discussed above, and so it does not preclude its presence being a result of photo-oxidation. Similar to sample S113, while 
the distribution of $\mathrm{CdSO}_{4} \cdot n \mathrm{H}_{2} \mathrm{O}$ does not eliminate its possible role as a synthesis starting reagent, the high chloride concentration of this sample suggests that $\mathrm{CdCl}_{2}$ is more likely to have functioned in this role.

The zinc content of $\mathrm{S} 117$ in comparison to S113 is also notable (see Fig. 3). Cadmium is mined from zinc ores, and therefore zinc is commonly observed in cadmium yellow paints in at least trace amounts (with Zn substituted for Cd on the CdS crystal lattice). The distribution of zinc observed in S117, however, suggests that zinc white $(\mathrm{ZnO})$ may have been added to this paint as a physical mixture to create a paler shade of cadmium yellow. The homogeneous distribution of zinc in the paint suggests that the addition was made by the manufacturer of the paint rather than by Matisse. Alternately, cadmium zinc sulphides were developed in the early $20^{\text {th }}$ century to produce paler shades of cadmium yellow that were more stable with an overall formula $\mathrm{Cd}_{1-x} \mathrm{Zn}_{x} \mathrm{~S}$, and were first referred to in the paint chemistry literature in $1914 .^{38}$ The use of a pale yellow in this area would be consistent with the lighter shade of yellow that can be observed in Matisse's oil sketch of the work [Henri Matisse. Sketch for Le Bonheur de vivre (Joy of Life) 1905-1906 San Francisco Museum of Modern Art, 91.160].

In Fig. 7 the IR intensity images for cadmium oxalate for samples S113 and S117 are shown. These images were generated using the absorption band at $1314 \mathrm{~cm}^{-1}$ that corresponds to the $\mathrm{C}_{2} \mathrm{O}_{4}{ }^{2-}$ functional group with an integration region between 1296 and $1330 \mathrm{~cm}^{-1}$. Regions of interest are circled in red and blue/grey in each image, representing high and relatively low integrated signal intensity regions (so-called hot and cold spots) for this oxalate signature in each of the paint cross sections, respectively. The spectra for those regions are stacked in the accompanying graph. The $1314 \mathrm{~cm}^{-1}$ absorption band is clearly visible in the spectra from the two 'hot spot' regions of interest chosen, but in sample S117, even the 'cold spot' of the sample still contains some oxalate, as evidenced by the band in the average spectrum.
The cadmium oxalates are concentrated at or near the surface of the paint layer for both S113 and S117, demonstrating that this is a photo-oxidation product rather than a paint filler in the cadmium yellow paints from both regions of the painting. This data also confirms that once cadmium oxalate has formed the low solubility of this photo-degradation product prevents it from becoming distributed throughout the paint film. This distribution of oxalates at the surface is consistent with micro FTIR imaging of sample S5 from below the central reclining figures (see Pouyet et al. this volume). In fact, the micro FTIR imaging data shows a stratigraphy of photo-degradation products where oxalates are located at the very surface, followed by carbonates, and then sulphates. Such a stratigraphy appears to be replicated here, which can be seen in particular when the three maps for sample S117 are viewed together. This stratigraphy, alongside the high chloride levels of the paint samples, is suggestive of all three phases being related to photo-degradation, and their distribution being related to their respective solubilities. This demonstrates the importance of understanding the potential movement of soluble material (in particular degradation products) throughout oil paintings as has been pointed out by Keune et al. ${ }^{39}$

In Fig. 8, two stacks of spectra from single pixel line scans within the measurement area for samples S113 and S117 are shown (see Panels A and B). The black lines on the IR images of the cadmium carbonate distributions (similar to those shown in Panels B in Fig. 4 and 5) indicate the pixels that these spectra are extracted from. These lines have been chosen to display a series of spectra where there is substantial chemical variation visible, with varying contributions of the carbonate, sulphate and oxalate concentrations. The green, blue and red highlighted windows on the spectra show the absorption bands that we have used to visualize the concentrations of these individual components. These spectra, compared side by side (and with the same scale), suggest that sample S117 has a higher contribution from oxalate than sample S113 based on the intensity of
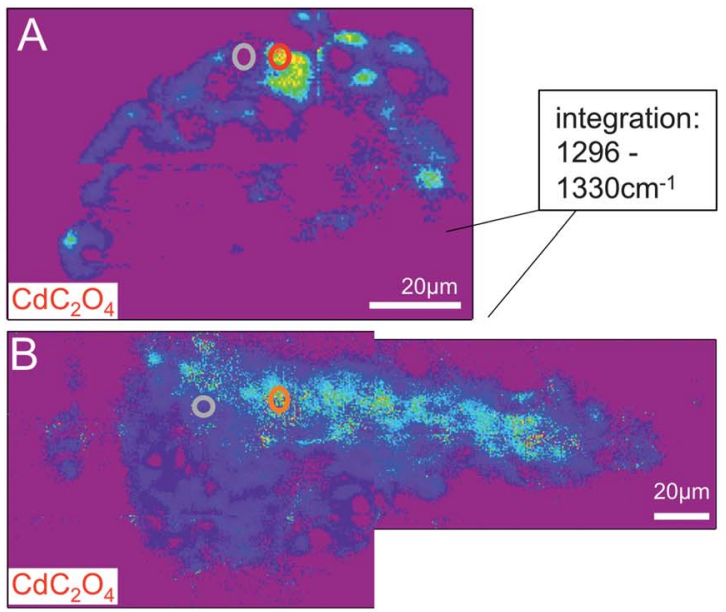

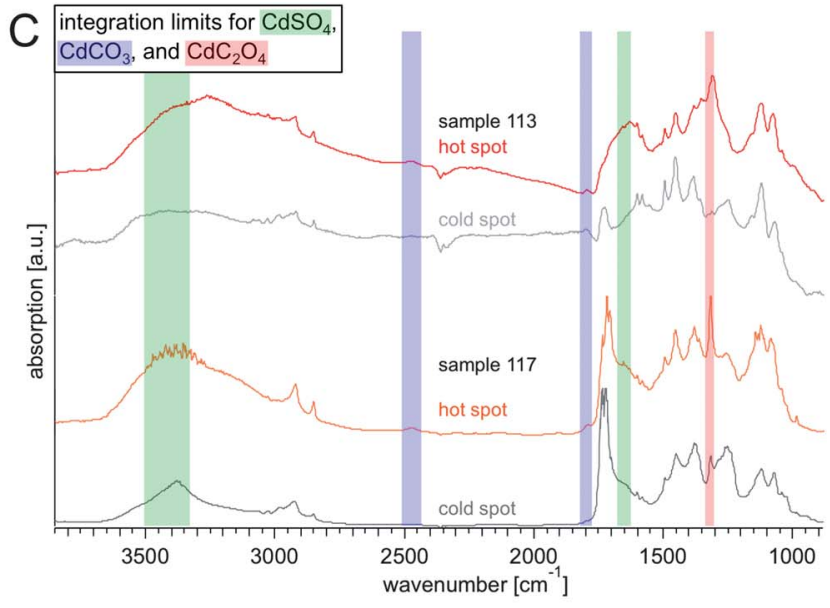

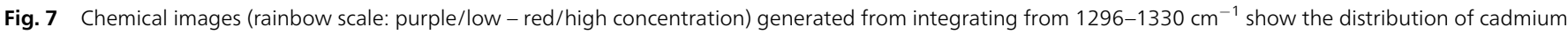

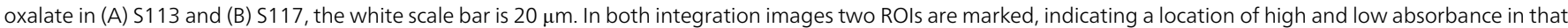

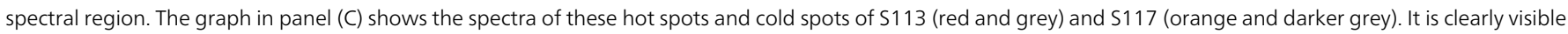
that the cadmium oxalate has a higher distribution towards the surface of the paint chips. 

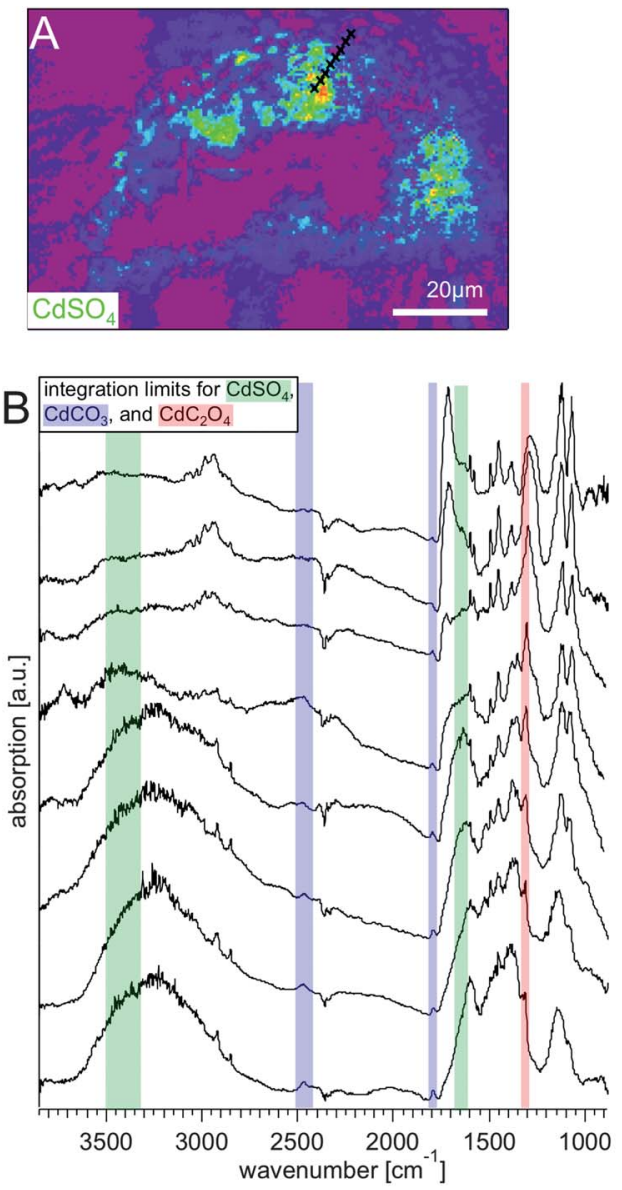
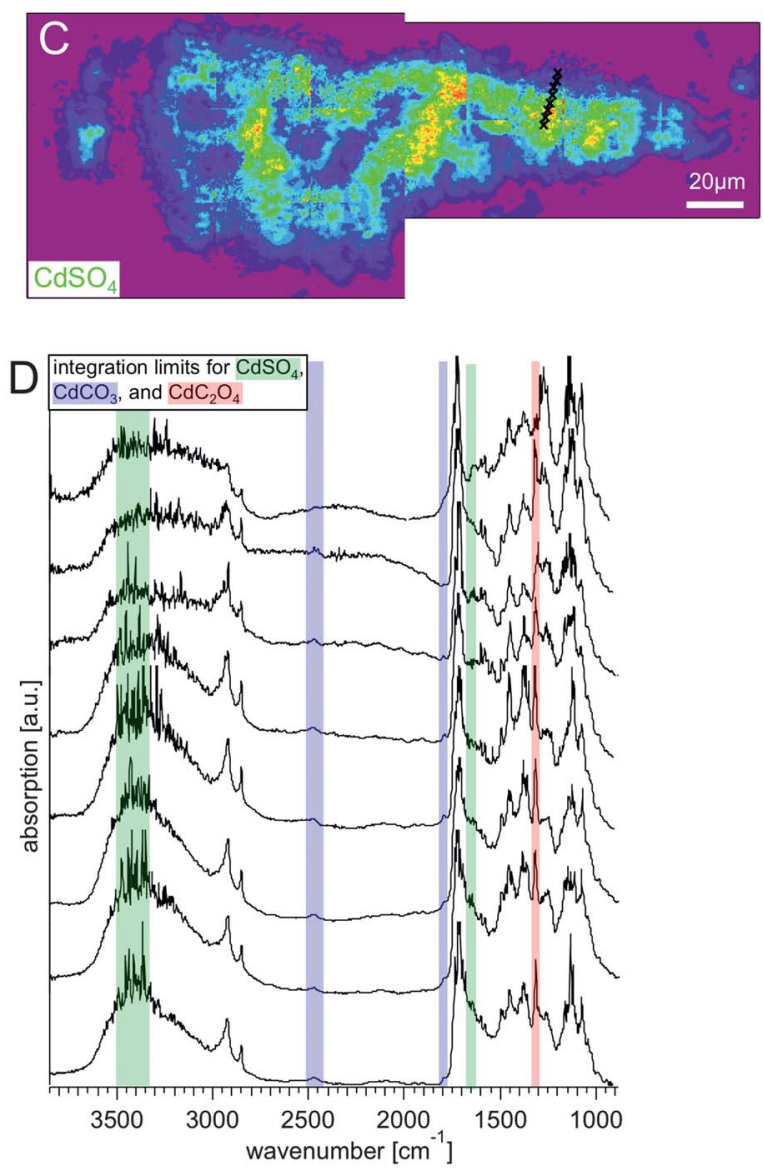

Fig. 8 Integration images of (A) S113 and (C) S117 between 3219 and $3612 \mathrm{~cm}^{-1}$, a region where only the sample absorbs as can be verified by looking at Fig. 4 . The white scale bars are $20 \mu \mathrm{m}$. The black crosses along the lines starting at the surface of the paint layer and transiting to the interior layers, mark the spots at which single pixel spectra have been extracted. These spectra are plotted in the graphs in panel (C) for S113 and (D) for S117. The complexity of the spectra and the variation of the distribution of the components can be seen in detail.

the band at $1314 \mathrm{~cm}^{-1}$. Sample S113, on the other hand, appears to have a higher concentration of carbonate based on the intensity of the band at $1777-1816 \mathrm{~cm}^{-1}$.

\section{Conclusions}

IRENI SR-FTIR imaging has been used to map the mid-IR active photo-degradation phases in two thin sections of cadmium yellow paint removed from Henri Matisse's Le Bonheur de vivre (1905-1906, The Barnes Foundation). The altered cadmium yellow paints from the darkened yellow foliage at the upper left of the work and the lightened yellow field beneath the central reclining figures both contain cadmium carbonate, cadmium sulphate, and cadmium oxalate. Each of these phases was imaged to probe its role in the cadmium yellow synthesis, formulation, and photo-degradation mechanisms. It was established that cadmium oxalate is localized at the surface of the paint layer, cadmium carbonate is also found deeper in the layer but still enriched at the surface, and cadmium sulphate is distributed throughout the paint layer. This distribution, along with the chloride content of the paint (which suggests a cadmium chloride starting material) is consistent with an alteration mechanism in which the cadmium sulphide is oxidized directly to form cadmium sulphate, which is then converted to cadmium carbonate and cadmium oxalate. A closely related stratigraphy of cadmium yellow photo-degradation products has recently been observed for Vincent van Gogh's Flowers in a Blue Vase (1887 (ref. 1)), in which the oxalate is thought to stem from degradation of the painting's varnish, and in a second sample from beneath the Le Bonheur de vivre's central reclining Fig. (S5)† discussed elsewhere in this volume (see Pouyet et al.).

The relative solubilities of the three photo-degradation products are also relevant to their locations in the paint film. It is expected that in a high relative humidity environment that even if cadmium sulphate were forming at the surface of the paint film as a result of photo-oxidation, that this soluble salt could become distributed throughout the paint layer during years of relative humidity cycling. The lower solubilities of cadmium oxalate and cadmium carbonate would contribute to their remaining at the surface after formation to create an alteration layer. Both of these phases are white (as is cadmium sulphate), and so could be expected to contribute to the formation of a lightened alteration crust such as the ivory crusts observed on Le Bonheur de vivre below the central reclining 
figures. The darker colour of the alteration layer at the upper left can be observed in cross-section as well as macroscopically, but it does not result from the distribution of the major mid-IR active photo-degradation phases observed here.

\section{Acknowledgements}

J. Mass is grateful to the Winterthur Museum, the University of Delaware, the Lenfest Foundation, and the Mellon Foundation for their support of this research. A portion of this work was also supported by the Getty Conservation Institute. IRENI was developed under the grant MRI-DMR-0619759. This work was supported by the NSF under grant CHE-1112433. The SRC is funded by UW-Madison and UW-Milwaukee.

\section{Notes and references}

1 G. Van der Snickt, K. Janssens, J. Dik, W. De Nolf, F. Vanmeert, J. Jaroszewicz, M. Cotte, G. Falkenberg and L. Van der Loeff, Anal. Chem., 2012, 84, 10221-10228.

2 M. Cotte, J. Susini, J. Dik and K. Janssens, Acc. Chem. Res., 2010, 43, 705-714.

3 J. L. Mass, R. Opila, B. Buckley, M. Cotte, J. Church and A. Mehta, Appl. Phys. A: Mater. Sci. Process., 2013, 111, 59-68.

4 L. Bertrand, M. Cotte, M. Stampanoni, M. Thoury, F. Marone and S. Schoder, Phys. Rep., 2012, 519(2), 51-96.

5 M. Cotte, P. Dumas, Y. Taniguchi, E. Checroun, P. Walter and J. Susini, C. R. Phys., 2009, 10, 590-600.

6 J. P. Echard, M. Cotte, E. Dooryhee and L. Bertrand, Appl. Phys. A: Mater. Sci. Process., 2008, 92, 77-81.

7 M. Cotte, P. Dumas, G. Richard, R. Breniaux and P. Walter, Anal. Chim. Acta, 2005, 553, 105-110.

8 L. Zanella, F. Casadio, K. A. Gray, R. Warta, Q. Ma and J. F. Gaillard, J. Anal. At. Spectrom., 2011, 26, 1090-1097.

9 L. Robinet, M. Spring, S. Pages-Camagna, D. Vantelon and N. Trcera, Anal. Chem., 2011, 83, 5145-5152.

10 L. Cartechini, C. Miliani, B. G. Brunetti, A. Sgamellotti, C. Altavilla, E. Ciliberto and F. Dacapito, Appl. Phys. A: Mater. Sci. Process., 2008, 92, 243-250.

11 M. O. Figueiredo, T. P. Silva and J. P. Veiga, Appl. Phys. A: Mater. Sci. Process., 2010, 99, 357-361.

12 L. Zanella, F. Casadio, K. A. Gray, R. Warta, Q. Ma and J.-F. Gaillard, J. Anal. At. Spectrom., 2011, 26, 1090-1097.

13 N. Salvadó, S. Butí, J. Nicholson, H. Emerich, A. Labrador and T. Pradell, Talanta, 2009, 79, 419-428.

14 J. L. Mass, R. Opila, B. Buckley, M. Cotte, J. Church and A. Mehta, Appl. Phys. A: Mater. Sci. Process., 2013, 111, 59-68.

15 A. Lluveras, S. Boularand, A. Andreotti and M. Vendrell-Saz, Appl. Phys. A: Mater. Sci. Process., 2010, 99, 363-375.

16 M. J. Nasse, M. J. Walsh, E. C. Mattson, R. Reininger, A. Kajdacsy-Balla, V. Macias, R. Bhargava and C. J. Hirschmugl, Nat. Methods, 2011, DOI: 10.1038/ nmeth.2596.

17 M. J. Nasse, E. C. Mattson, R. Reininger, T. Kubala, S. Janowski, Z. El-Bayyari and C. J. Hirschmugl, Nucl. Instrum. Methods Phys. Res., Sect. A, 2011, 649, 172-176.
18 B. Leone, A. Burnstock, C. Jones, P. Hallebeek, J. Boon and K. Keune, ICOM Committee For Conservation 14th Triennial Meeting, The Hague, 2005.

19 B. Topalova-Casadiego and U. Plahter, The National Gallery Technical Bulletin 30th Anniversary Conference, London, 2011.

20 G. V. D. Snickt, J. Dik, M. Cotte, K. Janssens, J. Jaroszewicz, W. D. Nolf, J. Groenwegen and L. v. d. Loeff, Anal. Chem., 2009, 81, 2600-2610.

21 I. Fiedler and M. A. Bayard, in Artists' Pigments: A Handbook of Their History and Characteristics, ed. R. L. Feller, Oxford University Press, New York, 1986, vol. 1, pp. 65-108.

22 E. Pouyet, B. Fayard, C. Gervais, J. Kieffer, A. LluverasTenorio, J. Mass, F. Meirer, A. Nevin, M. Salome, D. Saviello, F. Sette, J. Susini, Y. Taniguchi and M. Cotte, Analyst, 2013.

23 C. S. Patterson, D. Carson, A. Phenix, H. Khanjian, K. Trentelman, J. Mass and C. Hirschmugl, e-Preserv. Sci., 2013, 10, 1-9.

24 E. C. Mattson, M. J. Nasse, K. Gough and C. J. Hirschmugl, Anal. Chem., 2012, 84, 6173-6180.

25 G. Osmond, J. J. Boon, L. Puskar and J. Drennan, Appl. Spectrosc., 2012, 66, 1136-1144.

26 J. van der Weerd, A. van Loon and J. J. Boon, Stud. Conserv., 2005, 50, 3-22.

27 K. Keune and J. J. Boon, Stud. Conserv., 2007, 52, 161-176.

28 A. van Loon and J. J. Boon, Spectrochim. Acta, Part B, 2004, 59, 1601-1609.

29 H. Amrania, A. P. McCrow, M. R. Matthews, S. G. Kazarian, M. K. Kuimova and C. C. Phillips, Chem. Sci., 2011, 2, 107111.

30 L. Monico, G. Van der Snickt, K. Janssens, W. De Nolf, C. Miliani, J. Verbeeck, H. Tian, H. Y. Tan, J. Dik, M. Radepont and M. Cotte, Anal. Chem., 2011, 83, 12141223.

31 L. Monico, K. Janssens, C. Miliani, B. G. Brunetti, M. Vagnini, F. Vanmeert, G. Falkenberg, A. Abakumov, Y. G. Lu, H. Tian, J. Verbeeck, M. Radepont, M. Cotte, E. Hendriks, M. Geldof, L. van der Loeff, J. Salvant and M. Menu, Anal. Chem., 2013, 85, 851-859.

32 L. Monico, K. Janssens, C. Miliani, G. Van der Snickt, B. G. Brunetti, M. C. Guidi, M. Radepont and M. Cotte, Anal. Chem., 2013, 85, 860-867.

33 L. Monico, G. Van der Snickt, K. Janssens, W. De Nolf, C. Miliani, J. Dik, M. Radepont, E. Hendriks, M. Geldof and M. Cotte, Anal. Chem., 2011, 83, 1224-1231.

34 F. Casadio, S. Xie, S. C. Rukes, B. Myers, K. A. Gray, R. Warta and I. Fiedler, Anal. Bioanal. Chem., 2011, 399, 2909-2920.

35 V. B. Sanap and B. H. Pawar, Chalcogenide Lett. , 2010, 7, 227231.

36 A. M. E. Raj, D. D. Jayanthi and V. B. Jothy, Solid State Sci., 2008, 10, 557-562.

37 K. Keune and J. J. Boon, ICOM-CC Lisbon Proceedings, Lisbon, 2011.

38 A. P. Laurie, The Pigments and Mediums of the Old Masters, Macmillan, London, 1914.

39 K. Keune and J. J. Boon, ICOM-CC 16th Triennial Conference, Lisbon, 2011. 